15.2

\title{
Особенности динамики температурного отклика сегнетоэлектрической керамики при исследовании электрокалорического эффекта
}

\author{
(С) Г.Ю. Сотникова, Г.А. Гаврилов, А.А. Капралов, Р.С. Пассет, Е.П. Смирнова \\ Физико-технический институт им. А.Ф. Иофффе РАН, Санкт-Петербург, Россия \\ E-mail: g.sotnikova@mail.ioffe.ru
}

Поступило в Редакцию 3 августа 2021 г.

В окончательной редакции 3 августа 2021 г.

Принято к публикации 14 сентября 2021 г.

\begin{abstract}
Определение температурного отклика образца на внешнее электрическое поле является основным способом изучения электрокалорического эффекта в сегнетоэлектриках. На примере образца твердого раствора $0.65 \mathrm{PbFe}_{2 / 3} \mathrm{~W}_{1 / 3} \mathrm{O}_{3}-0.35 \mathrm{PbTiO}_{3}$ показано, что при увеличении напряженности электрического поля в образце может реализоваться эффект шнурования тока, приводящий к образованию локальных областей повышенной проводимости. В силу малого объема шнура связанные с ним тепловые эффекты обладают малыми характерными временами, сравнимыми с временами электрокалорического отклика образца, и могут приводить к значительным ошибкам при детектировании электрокалорического эффекта.
\end{abstract}

Ключевые слова: электрокалорический эффект, температурный отклик, сегнетоэлектрическая керамика, эффект шнурования тока.

DOI: 10.21883/PJTF.2021.24.51795.18982

Последние годы отмечены растущим интересом к изучению электрокалорического эффекта (ЕСЕ) в сегнетоэлектриках, который рассматривается как один из новых подходов для разработки эффективных накопителей энергии и твердотельных охлаждающих устройств [1-4]. Анализ динамики электрокалорического отклика образца в широком диапазоне изменения амплитуд, форм и длительностей воздействия электрического поля представляет значительный фундаментальный и прикладной интерес, так как позволяет получить новую информацию о поляризационных процессах в различных материалах и оценить перспективы их практического использования. Повышение метрологической достоверности измерения количественных характеристик ЕСE, а именно электрокалорических разности температур $(\delta T)$ и коэффициента $e=\delta T / \Delta E$ (здесь $\Delta E$ - диапазон изменения электрического поля), остается актуальной экспериментальной задачей наряду с определением пробивной напряженности электрического поля $E_{b d}$. Величина реально достижимого значения $\Delta E$, не приводящего к пробою, может стать основным фактором, ограничивающим возможность практического применения ЕСЕ в силу соотношения $\delta T \sim \Delta E \leqslant E_{b d}$. Однако в опубликованных работах по исследованию ЕСЕ вопросам изменения проводимости материалов при приложении сильного внешнего электрического поля и связанным с этим тепловым эффектам, ограничивающим реально достижимую величину $\delta T$, уделяется незаслуженно мало внимания. Эта проблема неразрывно связана с общей задачей исследования материалов при воздействии электрических полей, решение которой во многом определяет перспективы создания и развития электронной компонентной базы на основе полупроводниковых и диэлектрических материалов [5-10]. Помимо развития теории необратимого „объемного“ теплового пробоя, вызванного потребностью предсказывать поведение изоляционных материалов в сильных электрических полях [5], большой интерес вызывает изучение ,локальных“ явлений, связанных с наличием различного вида дефектов на поверхности и в объеме полупроводников, приводящих к локальному увеличению плотности тока при приложении поля и соответственно локальному разогреву образцов: микроплазменный пробой [6], эффект „шнурования тока“ $[7,8]$. Эти исследования, пик которых пришелся на 60-80-е годы прошлого столетия и был связан с бурным развитием полупроводниковой элементной базы, не потеряли своей актуальности. В настоящее время возросший интерес к этим исследованиям связан с активным поиском новых эффективных материалов для солнечной энергетики [9], для электромеханических преобразователей на основе сегнетоэлектрических полимеров, монокристаллов и керамики различного состава, преимуществами которой являются легкость изготовления, прочность, стабильность, возможность получения сложных конфигураций [10].

Явление, аналогичное микроплазменному пробою в полупроводниках, описано нами в работе [11], где на примере исследования партии образцов многослойных структур сегнетоэлектрической керамики (релаксора) $0.55 \mathrm{~Pb}-\mathrm{Mg}_{1 / 3} \mathrm{Nb}_{2 / 3} \mathrm{O}_{3}-0.45 \mathrm{PbSc}_{1 / 2} \mathrm{Nb}_{1 / 2} \mathrm{O}_{3}$ было показано, что при значениях $E \sim(1 / 2) E_{b d}\left(E_{b d}-\right.$ ожидаемое значение пробойного поля) на фоне электрокалорического отклика образцов возникают явления, проявляющиеся в виде резких выбросов температуры. Получен- 


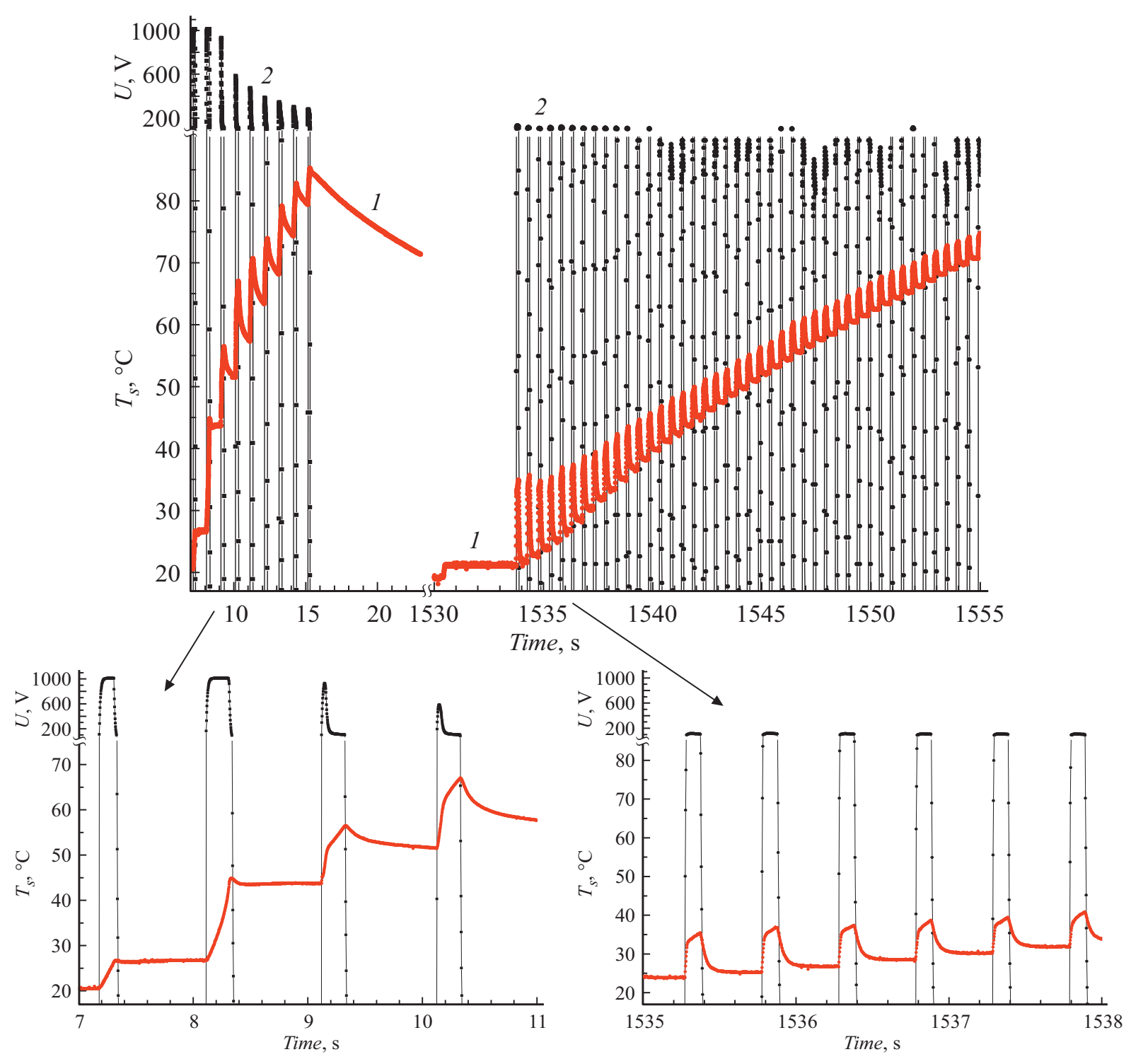

Рис. 1. Экспериментальные эпюры синхронного мониторинга температурного отклика $T_{s}$ (кривая 1 ) и напряжения $U$ (кривая 2) на образце сегнетоэлектрической керамики твердого раствора $0.65 \mathrm{PbFe}_{2 / 3} \mathrm{~W}_{1 / 3} \mathrm{O}_{3}-0.35 \mathrm{PbTiO}_{3}$ (диаметр $10 \mathrm{~mm}$, толщина $0.92 \mathrm{~mm}$ ) при приложении к нему последовательности прямоугольных импульсов напряжения амплитудой $U=1000 \mathrm{~V}$. В нижней части рисунка приведены участки эпюр, отражающие разный характер тепловых эффектов в образце, вызванных объемной проводимостью образца и эффектом шнурования тока.

ный эффект хорошо описывается в рамках модели частичного разряда в диэлектриках [12], искажает динамику температурного отклика образца и может приводить к завышению экспериментальных значений $\delta T$.

В настоящей работе нами представлены результаты исследования модельного объекта - сегнетоэлектрического твердого раствора $0.65 \mathrm{PbFe}_{2 / 3} \mathrm{~W}_{1 / 3} \mathrm{O}_{3}-0.35 \mathrm{PbTiO}_{3}$ (PFW-PT), расположенного вблизи морфотропной фазовой границы (МФГ) [13]. Наличие МФГ у твердых растворов является характерной особенностью, а также существенным требованием для получения высоких электромеханических и диэлектрических характеристик и, как показывают экспериментальные данные, перспективных электрокалорических свойств [14].
В экспериментах использовались образцы сегнетоэлектрической керамики $\mathrm{PFW}-\mathrm{PT}$ в виде таблеток толщиной $d \sim 0.3-1 \mathrm{~mm}$, диаметром $D \sim 10 \mathrm{~mm}$ с возженными серебряными электродами. Исследование температурного отклика образца на приложение электрического поля (прямой метод измерения $\delta T$ при изучении ЕСЕ) проводилось с использованием многоканальной экспериментальной установки, методик и алгоритмов прямых измерений пиро- и электрокалорического эффектов, опубликованных в [15].

На рис. 1 приведены результаты мониторинга температурного отклика образца толщиной $d=0.92 \mathrm{~mm}$ при приложении к нему последовательности прямоугольных импульсов напряжения с периодом $400 \mathrm{~ms}$ и 
длительностью 100-200 ms при амплитуде $U=1000 \mathrm{~V}$ $(E \sim 10 \mathrm{kV} / \mathrm{cm})$, устанавливаемой на выходе высоковольтного источника напряжения TREK609E-6. Значение $E \ll E_{b d} \approx 30 \mathrm{kV} / \mathrm{cm}$, где $E_{b d}$ - ожидаемая величина пробивной напряженности для данного образца (диэлектрическая проницаемость $\varepsilon \sim 10000$ в области комнатной температуры [13]), рассчитанная в соответствии с [16]. Одновременно с бесконтактным контролем температуры поверхности образца (кривая 1 на рис. 1) осуществлялся контроль напряжения (кривая 2 на рис. 1) непосредственно на образце с временни́м разрешением $2 \mathrm{~ms}$. Из рис. 1 видно, что в течение первых импульсов поля (до 9s) наблюдается линейный разогрев образца, характерный для джоулевого нагрева „объемным“ током проводимости, присущим данному образцу с известными значениями плотности $(\rho)$, теплоемкости $(c)$ и удельной проводимости $(\sigma(T))$. Заметим, что в диапазоне температур $20-110^{\circ} \mathrm{C}$ зависимость $\sigma(T)$ носит экспоненциальный характер [17]. После 9-й секунды эксперимента наблюдается изменение характера температурного отклика, сопровождаемое падением напряжения на образце, обусловленным предельной величиной тока используемого источника. Участок температурного отклика после выключения импульсов поля (после 15-й секунды эксперимента, рис. 1) позволяет определить постоянную времени тепловой релаксации образца $\tau_{0}=2 H / \rho c d \approx 30 \mathrm{~s}$ с учетом реальных условий теплообмена, определяемых коэффициентом теплообмена $(H)$ в условиях конкретного эксперимента (см. методику в [15]). При последующей подаче импульсов высокого напряжения $(t>1530 \mathrm{~s}$, рис. 1$)$ температурный отклик образца имеет вид обратимых тепловых эффектов с временами релаксации $\tau_{o n}$ (включение), $\tau_{o f f}$ (выключение) $\ll \tau_{0}$, при этом амплитуда импульсов напряжения не соответствует установленному значению $U=1000 \mathrm{~V}$ (рис. 1), а ограничивается уровнем $U=200 \mathrm{~V}$. Такая динамика температурного отклика образца PFW-PT хорошо согласуется с моделью обратимого электроннотеплового пробоя, разработанной ранее для полупроводниковых материалов и вызванной эффектом шнурования тока $[7,8]$. Как следует из [8], характерные времена таких тепловых процессов составляют $\tau_{o n} \sim 10^{-6}-10^{-10} \mathrm{~s}$ для полупроводниковых структур толщиной $d \sim 1 \mu \mathrm{m}$ связаны с малым объемом шнура, а небольшое затягивание $\tau_{o f f}>\tau_{o n}$ обусловлено наложением двух тепловых процессов: быстрое выключение шнура тока и медленное остывание нагретого объема образца в целом.

Результаты, представленные на рис. 2, позволяют рассчитать количественные параметры регистрируемого теплового эффекта в исследуемом образце: постоянные времени $\tau_{o n}=15 \mathrm{~ms}, \tau_{o f f}=40 \mathrm{~ms}$ и амплитуду $d T=10^{\circ} \mathrm{C}$ при температуре образца вблизи комнатной. Значение $\tau_{o n}=15 \mathrm{~ms}$ хорошо согласуется с характерными временами электрокалорического отклика образцов аналогичного размера сегнетоэлектрической керамики различного состава, полученными экспериментально в [15]. В то же время регистрируемая амплитуда

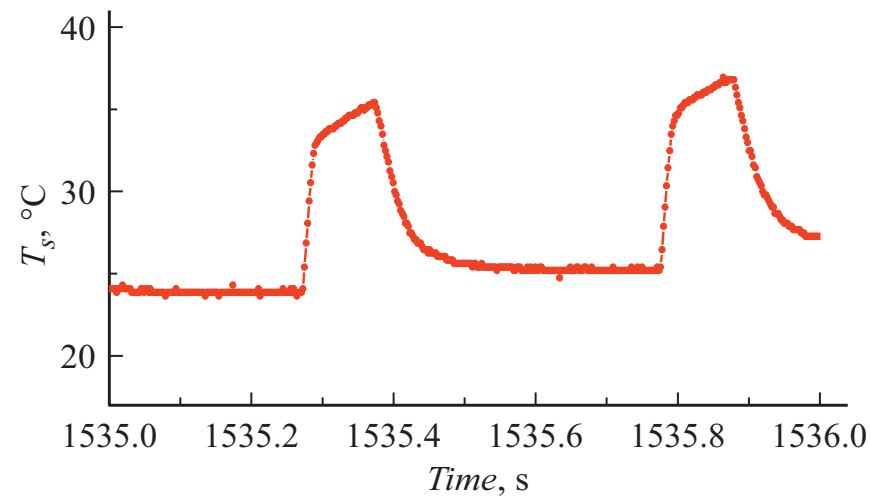

Рис. 2. Температурный отклик образца сегнетоэлектрической керамики твердого раствора $0.65 \mathrm{PbFe}_{2 / 3} \mathrm{~W}_{1 / 3} \mathrm{O}_{3}-0.35 \mathrm{PbTiO}_{3}$, демонстрирующий обратимый электронно-тепловой эффект в режиме шнурования тока.

температурного отклика $d T=10^{\circ} \mathrm{C}$ значительно превосходит типичные значения электрокалорической разности температур $\delta T \sim 1-2^{\circ} \mathrm{C}$, достигнутой на данный момент в объемных керамических образцах различного состава $[1-4]$.

В настоящей работе впервые экспериментально зарегистрирован обратимый тепловой эффект, наблюдаемый для сегнетоэлектрического твердого раствоpa $0.65 \mathrm{PbFe}_{2 / 3} \mathrm{~W}_{1 / 3} \mathrm{O}_{3}-0.35 \mathrm{PbTiO}_{3}$, расположенного на МФГ, который может быть объяснен в рамках модели обратимого электронно-теплового пробоя, связанного с эффектом шнурования тока. Полученные значения постоянных времени нагрева и охлаждения сопоставимы с характерными временами электрокалорического отклика керамических материалов.

Выявленные особенности динамики температурного отклика материала во внешних электрических полях имеют особое значение при исследованиях ЕСЕ, требующих приложения переменного электрического поля с максимально возможной амплитудой, не приводящей к пробоям образца. Неконтролируемое возникновение „аномалий“ температурного отклика образца сегнетоэлектрической керамики на воздействие внешнего электрического поля, связанных с локальными изменениями его проводимости, может привести к значительным ошибкам при регистрации ЕСЕ в новых материалах и завышению $\delta T$ при численном моделировании ЕСЕ без учета реальной величины допустимого изменения рабочего напряжения. Избежать таких ошибок можно только при одновременном контроле динамики изменения температуры и напряжения на исследуемом образце с высоким временны́м разрешением, а также условий теплообмена, определяющих истинную величину постоянной времени тепловой релаксации образца в целом.

В работе продемонстрирована актуальность комплексного изучения закономерностей и механизмов объемного и локального изменения проводимости сегнетоэлектрических материалов, которое представляет значи- 
тельный фундаментальный и практический интерес, что стимулирует дальнейшие исследования.

\section{Благодарности}

Авторы выражают благодарность М.Е. Левинштейну за плодотворные дискуссии об электронно-тепловых механизмах пробоя в полупроводниках.

\section{Финансирование работы}

Работа выполнена в рамках госзадания (научные темы 0040-2019-0019, 0040-2019-0031).

\section{Конфликт интересов}

Авторы заявляют, что у них нет конфликта интересов.

\section{Список литературы}

[1] Y. Liu, J.F. Scott, B. Dkhil, Appl. Phys. Rev., 3, 031102 (2016). DOI: $10.1063 / 1.4958327$

[2] S. Pandya, J. Wilbur, J. Kim, R. Gao, A. Dasgupta, C. Dames, L.W. Martin., Nature Mater., 17, 432 (2018). DOI: $10.1038 / \mathrm{s} 41563-018-0059-8$

[3] T. Zhang, X.-S. Qian, H. Gu, Y. Hou, Q.M. Zhang, Appl. Phys. Lett., 110, 243503 (2017). DOI: 10.1063/1.4986508

[4] Electrocaloric materials: new generation of cooler, ed. by T. Correia, Q. Zhang (Springer, 2013). DOI: $10.1007 / 978-3-642-40264-7$

[5] Г.А. Воробьев, Ю.П. Похолков, Ю.Д. Королев, В.И. Меркулов, Физика диэлектриков (область сильных полей) (Изд-во ТПУ, Томск, 2003).

[6] И.В. Грехов, Ю.Н. Сережкин, Лавинный пробой $p-n$ переходов в полупроводниках (Энергия, Л., 1980).

[7] В.В. Пасынков, Л.С. Чиркин, Полупроводниковые прибоpы (Высш. шк., М., 1987).

[8] Электронные явления в халькогенидных стеклообразHblx полупроводниках, под ред. К.Д. Цэндина (Наука, СПб., 1996), с. 224-299.

[9] K.A.K. Niazi, W. Akhtar, H.A. Khan, Y. Yang, S. Athar, Solar Energy, 190, 34 (2019). DOI: 10.1016/j.solener.2019.07.063

[10] М. Лайнс, А. Гласс, Сегнетоэлектрики и родственные им материаль (Мир, М., 1981).

[11] Г.Ю. Сотникова, Г.А. Гаврилов, А.А. Капралов, Р.С. Пассет, Е.П. Смирнова, ФТТ, 62 (10), 1631 (2020). DOI: 10.21883 /FTT.2020.10.49911.099

[12] M. Refaey, A.A. Hossam-Eldin, T. Negm, in 18th IEEE Int. Middle East Power Systems Conf. (MEPCON) (Helwan, Egypt, 2016). DOI: 10.1109/MEPCON.2016.7836959

[13] E. Smirnova, A. Sotnikov, M. Shevelko, N. Zaitseva, H. Schmidt, J. Mater. Sci., 56, 4753 (2021). DOI: $10.1007 / \mathrm{s} 10853-020-05613-3$

[14] Y. Bai, D. Wei, L.-J. Qiao, Appl. Phys. Lett., 107, 192904 (2015). DOI: $10.1063 / 1.4935424$

[15] G.Yu. Sotnikova, G.A. Gavrilov, A.A. Kapralov, K.L. Muratikov, E.P. Smirnova, Rev. Sci. Instrum., 91, 015119 (2020). DOI: $10.1063 / 1.5108639$

[16] C. Neusel, G.A. Schneider, J. Mech. Phys. Solids, 63, 201 (2014). DOI: 10.1016/j.jmps.2013.09.009
[17] Г.Ю. Сотникова, Г.А. Гаврилов, К.Л. Муратиков, Р.С. Пассет, Е.П. Смирнова, ФТТ, 63 (6), 730 (2021). DOI: 10.21883 /FTT.2021.06.50930.024 